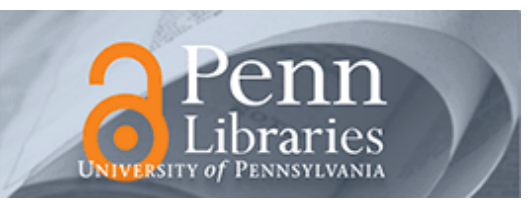

University of Pennsylvania

ScholarlyCommons

May 2002

\title{
Tonal Behavior Analysis of an Adaptive Second-Order Sigma-Delta Modulator
}

Xiaohong Sun

Philips Research

Kenneth R. Laker

University of Pennsylvania, laker@seas.upenn.edu

Follow this and additional works at: https://repository.upenn.edu/ese_papers

\section{Recommended Citation}

Xiaohong Sun and Kenneth R. Laker, "Tonal Behavior Analysis of an Adaptive Second-Order Sigma-Delta Modulator", . May 2002.

Copyright 2002 IEEE. Reprinted from Proceedings of the IEEE International Symposium on Circuits and Systems 2002 (ISCAS 2002), Volume 4, pages IV-277 - IV-280.

Publisher URL: http://ieeexplore.ieee.org/xpl/tocresult.jsp?isNumber=21779\&page=4

This material is posted here with permission of the IEEE. Such permission of the IEEE does not in any way imply IEEE endorsement of any of the University of Pennsylvania's products or services. Internal or personal use of this material is permitted. However, permission to reprint/republish this material for advertising or promotional purposes or for creating new collective works for resale or redistribution must be obtained from the IEEE by writing to pubs-permissions@ieee.org. By choosing to view this document, you agree to all provisions of the copyright laws protecting it.

This paper is posted at ScholarlyCommons. https://repository.upenn.edu/ese_papers/63

For more information, please contact repository@pobox.upenn.edu. 


\title{
Tonal Behavior Analysis of an Adaptive Second-Order Sigma-Delta Modulator
}

\begin{abstract}
This paper analyzes the tonal behavior of an adaptive second-order sigma-delta modulator, which was developed and published by the same authors. Idle channel tones, caused by non-white quantization error, is not desirable in applications where the human ear is the end receiver. Besides their relatively small magnitude tones in the baseband, most sigma-delta modulators produce high-powered tones near $f_{s} / 2$. It is a more serious problem because the clock noise near $f_{S} / 2$ can couple these tones down into the baseband. Various simulations show that the more randomized nature of the aforementioned adaptive architecture makes it more advantageous in tonal behavior, particularly attractive in that it significantly reduces the dominant tone near $f_{s} / 2$, which can not be reduced by dithering in a standard second order single-bit modulator. With comparison to the standard second-order sigma-delta modulators, the results are illustrated in both frequency and time domains.
\end{abstract}

\section{Comments}

Copyright 2002 IEEE. Reprinted from Proceedings of the IEEE International Symposium on Circuits and Systems 2002 (ISCAS 2002), Volume 4, pages IV-277 - IV-280.

Publisher URL: http://ieeexplore.ieee.org/xpl/tocresult.jsp?isNumber=21779\&page=4

This material is posted here with permission of the IEEE. Such permission of the IEEE does not in any way imply IEEE endorsement of any of the University of Pennsylvania's products or services. Internal or personal use of this material is permitted. However, permission to reprint/republish this material for advertising or promotional purposes or for creating new collective works for resale or redistribution must be obtained from the IEEE by writing to pubs-permissions@ieee.org. By choosing to view this document, you agree to all provisions of the copyright laws protecting it. 


\title{
Tonal Behavior Analysis of an Adaptive Second-Order Sigma-Delta Modulator
}

\author{
Xiaohong Sun ${ }^{*}$ and Kenneth R. Laker ${ }^{\star}$
}

\author{
'Systems and Electronic Design, Philips Research \\ 345 Scarborough Road, Briarcliff Manor, NY 10510, USA \\ 'Department of Electrical Engineering, University of Pennsylvania \\ 200 south 33rd Street, Philadelphia, PA 19104, USA
}

\begin{abstract}
This paper analyzes the tonal behavior of an adaptive second-order sigma-delta modulator, which was developed and published by the same authors. Idle channel tones, caused by non-white quantization error, is not desirable in applications where the human ear is the end receiver. Besides their relatively small magnitude tones in the baseband, most sigma-delta modulators produce highpowered tones near $f_{s} / 2$. It is a more serious problem because the clock noise near $f s / 2$ can couple these tones down into the baseband. Various simulations show that the more randomized nature of the aforementioned adaptive architecture makes it more advantageous in tonal behavior, particularly attractive in that it significantly reduces the dominant tone near $f_{s} / 2$, which can not be reduced by dithering in a standard second order single-bit modulator. With comparison to the standard second-order sigma-delta modulators, the results are illustrated in both frequency and time domains.
\end{abstract}

\section{INTRODUCTION}

Sigma-delta modulators $(\Sigma \Delta \mathrm{M})$ have been extensively used in quality audio applications due to their high resolution and relatively simple analog implementation [1]. However, the tonal behavior of most sigma-delta modulators for $\mathrm{dc}$ inputs, can be a problem in $A / D$ or $D / A$ conversions, especially when the end receiver is the human ear, which is very sensitive to certain coloration and periodicity in sound [2]. Idle channel tones, also referred to as pattern noise, are caused by non-white quantization noise, which is typical for sigma-delta modulators, in which one-bit quantizers are often chosen [3]. Various dithering techniques have been effective in whitening the pattern noise with different amounts of degradation in dynamic range [4].

While the baseband tones have relatively small magnitudes, the tones near $f s / 2$ contain much higher power. It is more serious a problem because even a small amount of clock noise near $f / 2$ can couple these tones down to the baseband. These high frequency tones of a standard second-order single-bit sigma-delta modulator can be more troublesome because they do not respond to dithering [2]. Nevertheless, the unique architecture with Adaptive Integrator Bounding (AIB) in [5] shows significant reduction in the dominant tone near $f s / 2$, which can greatly degrade the performance of an audio system when coupled down to the baseband. Extensive simulations of various inputs have been done to verify this statement, with the comparison to the standard normalized second-order structure. The results are shown in both frequency domain (power spectrum) and time domain (short-term autocorrelation.)

The paper is organized as follows: Section 2 reviews the AIB architecture and its contribution to whitening the quantization error, which is a benefit that comes with the main original advantage: the extended full-scale dynamic range. Section 3 analyzes the tonal behavior of the AIB architecture, focusing on the effect of the high-powered tones near $f s / 2$. Simulation conditions and results are included in Section 4 followed by the conclusion.

\section{The AIB ARCHITECTURE}

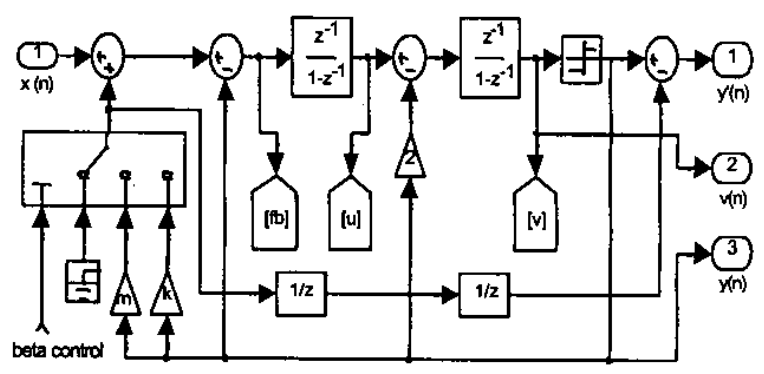

Figure 1 Second order $\Sigma \Delta M$ with AIB

The original idea of the AIB algorithm is to extend the practical dynamic range to full scale in a second order single-bit modulator, while the standard structure has 
dramatic premature reduction in SNR at large inputs. The degradation in the performance is caused by the overloading effect of the non-linear 1-bit quantizer, and it practically degrades the dynamic range from the full scale by $2 \sim 3 \mathrm{~dB}$. Shown in Figure 1 is a normalized second-order single-bit modulator with the AIB algorithm [6]. The detailed discussion of AIB can be found in $[5,6]$ and this paper mainly describes the advantages of the AIB architecture with emphasis on its more randomized nature, which leads to its contribution to whitening the auantizaiton error.

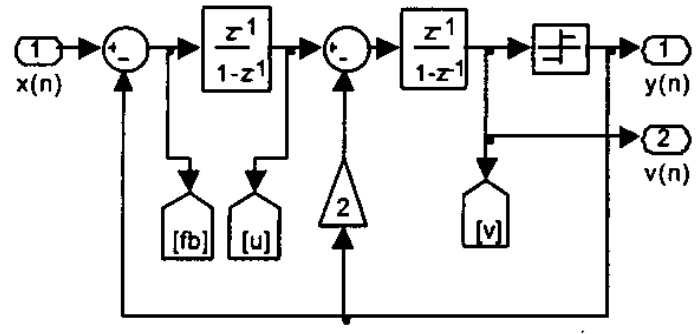

Figure 2 Standard Second-Order $\Sigma \Delta M$

The difference between the AIB structure and a standard second-order sigma-delta modulator (SSD), shown in Figure 2, is that the AIB architecture has an additional feedback coefficient $\beta(n)$ (stands for the signal 'beta control') through the multipoint switch, is added to the input node. The input of the first integrator $\mathrm{fb}(\mathrm{n})$ in the AIB scheme becomes

$$
f b(n)=x(n)+(\beta(n)-1) y(n)
$$

instead of the $\mathrm{fb}(\mathrm{n})$ in the SSD

$$
f b(n)=x(n)-y(n)
$$

The compensation is done in digital form at the output. It can be viewed as a pre- and post- processing appended to a SSD system without changing the functionality of the modulator, provided that the digital subtraction matches the analog addition [6].

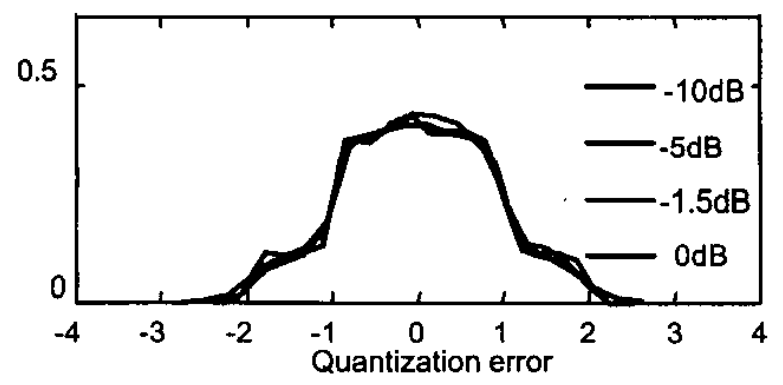

Figure 3 Prob. density of e(n) in AIB
By dynamically controlling the feedback coefficient of the first integrator, the AIB algorithm effectively bounds the upper limit of the integrator outputs, extends the nonclipping input level to its full scale. In addition to these advantages in general performance, it had been noticed in [5] that the AIB has more whitening quantization noise than that of the standard structure, and it will be discussed in the next section.

\section{TONAL ANALYSIS}

Idle channel tones or pattern noise, of which the spectrum contains discrete tones, noise, is caused by the non-white quantizaton noise. In spite of the fact that Bennet's theorem has been widely used in simplifying the system analysis of sigma-delta modulators, the additive white noise approximation of the non-linear 1-bit quantization is far from accurate [3]. To the contrary, the quantization error from the sigma-delta modulation is typically not white and usually depends on both the amplitudes and frequencies of the input signal. In terms the input dependency of the quantization error, the preliminary study on probability distribution in [5], shown in Figure 3 and Figure 4, provides an intuitive support of the earlier statement that the AIB has a more randomized nature and is less signal-dependent than the standard second-order sigma-delta modulator. This study is done by using a group of sinusoid inputs at same frequency but with different amplitudes [5].

For dc inputs, almost all single-stage sigma-delta modulators sound tonal. Early in the literature, there was suggestion in [7] that the analog second or higher order modulators will not sound tonal because the small thermal noises are enough to whiten the quantization noise. But more recent research has shown that the thermal noise is not enough to whiten the colored noise. Tones have been found even in the eighth-order modulators [2]. Various dithering methods have been adopted to accommodate different applications with the tradeoff being the dynamic range. Comparisons of these methods can be found in [4].

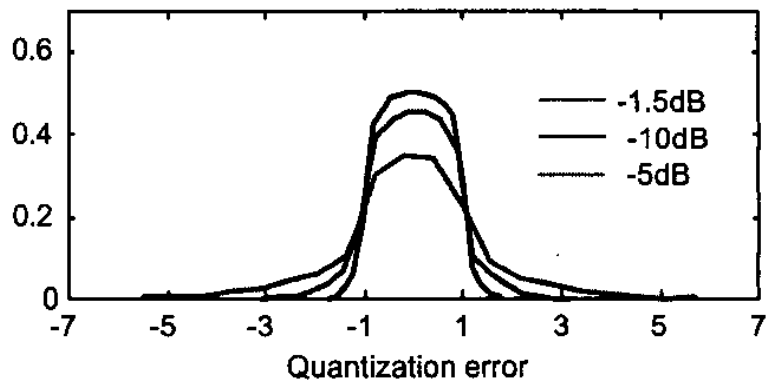

Figure 4 Prob. density of e(n) in SSD 
To locate the tone frequencies for a given dc input, the following empirical equation is normally used [2]. The tones are at

$$
f_{0}=\frac{n f_{s}\left|A_{d d}\right|}{2 \Delta} \quad n=\{0,1,2, \cdots\}
$$

where $f_{s}$ is the sampling frequency, $\left|\mathrm{A}_{\mathrm{dc}}\right|$ is the magnitude of the dc input level and $\Delta$ is the interval of the 1-bit quantizer. In consistency with the previous study, the simulation results in the next section also shown that the tone of $n=4$ is the dominant one and remains high above the noise floor while other baseband tones are whitened by the small amount of thermal noise added to the input [2].

While the tones in the lower frequencies are relatively small in magnitude, most sigma-delta modulators produce highpowered tones near $f_{s} / 2$ for nearly all classes of inputs. This problem is more serious because even the slightest amount of coupling of $f_{s} / 2$ into the baseband can potentially destroy the performance. Dithering does help to reduce these tones for modulators higher than second order, but the standard second-order 1-bit modulator showed hardly any reduction with the presence of dithering [2]. On the other hand, the AIB architecture has significantly reduced these tones by its own randomized nature. Particular, it practically eliminates the dominant tone near $f_{s} / 2$, which is typically $20 \sim 30 \mathrm{~dB}$ above the highest point of the noise floor. This alone will reduce the sensitivity of the aliasing effect by $20 \sim 30 \mathrm{~dB}$. It will also provides extra $20 \sim 30 \mathrm{~dB}$ isolation between the analog and digital sections at these frequencies in hardware implementations [2].

For tonal analyses, it has been well known that the power spectrum estimation alone is not sufficient to reveal the tones that is short-term periodic in time domain, while the human ear is sensitive to these tones. Therefore, the autocorrelation estimation has been extensively used in estimations of tonal behavior. For a finite-length real sequence, the discrete-time autocorrelation is given by

$$
\phi_{x x}(m)=\frac{1}{2 N} \sum_{n=-N}^{N-1} x(n) x(n+m)
$$

in which $\phi_{x}(0)$ is the mean-square value of the sequence, or its average power. The rest values of $\phi_{x x}(m)$ represents the inter-dependency of the signal to itself with $m$ shifts in the sequence. If the original sequence is aperiodic, $\phi_{\mathrm{xx}}(\mathrm{m})$ tends toward a constant when $m \neq 0$. If the original sequence is pure random (white) noise with zero mean, then $\phi_{x x}(m) \rightarrow 0$ when $m \neq 0$. Therefore, the advantages of the AIB design can also be shown by this effective time domain estimation, which is also included in the following section of simulation results.

\section{SIMULATION RESULTS}

This section includes the simulation results of both the frequency and time domain estimation of the AIB system. In the standard second order modulator, applying dithering trades in the dynamic range and lowers the peak SNR [2], but the AIB algorithm extends the dynamic range, improves the peak SNR while it whitens the quantization error. For the example in [5], it is about $2 \mathrm{~dB}$ extension in the dynamic range and $5 \mathrm{~dB}$ improvement in the peak $\mathrm{SNR}$, which is shown in Figure 5.
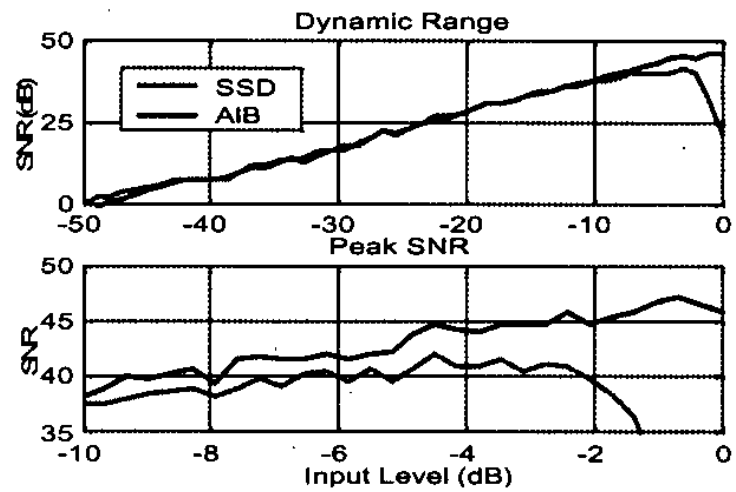

Figure 5 Dynamic range and peak SNR

More specific simulations are done using a dc input of $1 / 256(-48.16 \mathrm{~dB})$ to compare the tonal behavior of the standard structure and the AIB design. The sampling rate is chosen as $1.024 \mathrm{MHz}$ over a $4 \mathrm{KHz}$ speech band resulting in an oversampling ratio of 256 . A total power of $-95.32 \mathrm{~dB}$ input-referred thermal noise are added to the input and no dithering has been applied. This value is chosen to be below the ideal dynamic range, which is $94.22 \mathrm{~dB}$ according to the linear model definition [5],

$$
D R=\frac{3}{2}\left(\frac{2 L+1}{\pi^{2 L}}\right)\left(2^{N}-1\right)^{2} O S R^{2 L+1}
$$

in which $\mathrm{N}$ is the bit-wise quantization levels(for the singlebit quantizer, $\mathrm{N}=1$ ), $\mathrm{L}$ is the order of the modulator and $\mathrm{OSR}$ is the oversampling ratio. The thermal noise floor in a practical system is usually higher but this small value is used so that it is distinguished from non-subtractive dithering (at least $1 \mathrm{LSB}$ in general) at the input.

Figure 6 is the comparison of the power spectrum. Both systems contain tones at $n=4$, in this case, at $4 \mathrm{KHz}$, according to equation (3). The magnitude is about $-91 \mathrm{~dB}$. The spectra also demonstrate that the AIB does not have the dominant tone near $\mathrm{fs} / 2$, but the dominant tone of the standard structure is $-9.9 \mathrm{~dB}$, which is about $20 \mathrm{~dB}$ above the highest point of the noise floor. Assuming a coupling 
coefficient of $-60 \mathrm{~dB}$, this largest tone will be coupled down to the baseband of the magnitude of $-69.9 \mathrm{~dB}$, which will definitely destroy the performance of the modulator.

The short-term autocorrelation estimations of the modulator outputs are shown in Figure 7. The AIB is obviously advantageous in the time domain evaluation, which is considered more effective in tonal analysis [2]. There is still some periodicity in the AIB design, but it greatly outperforms the standard second-order modulator whose pure periodic characteristic is clearly revealed in Figure 7(a).

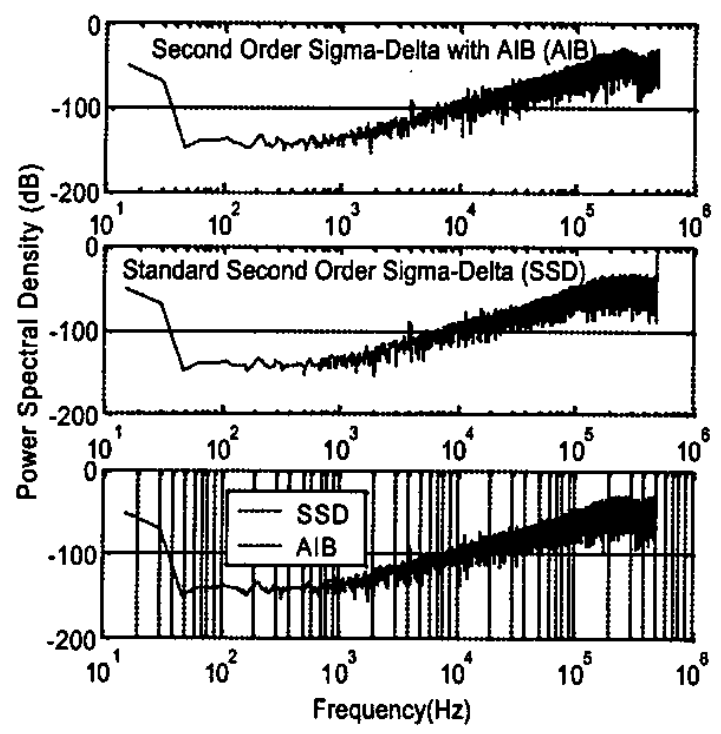

Figure 6 Power Spectrum Estimation
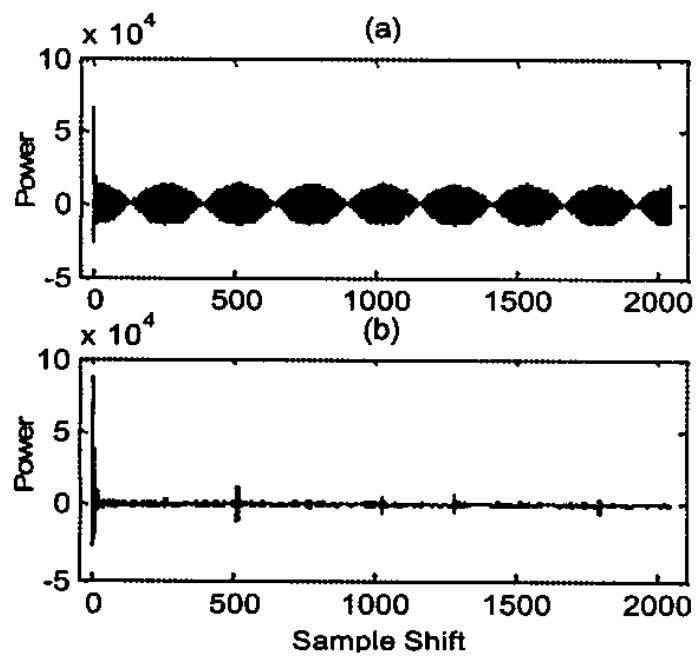

Figure 7 Autocorrelation estimation (a) Standard second-order (b) AIB

\section{CONCLUSION}

The tonal behavior of an adaptive second-order single-bit sigma-delta modulator (AIB) has been discussed and simulated, with the comparison of a standard second-order single-bit modulator.

The frequency domain estimations show that, the tonal behavior of the AIB and that of the standard second-order structure are similar in lower frequencies. But the AIB is almost tone-free at high frequencies near $f s / 2$, while the standard structure has a dominant tone of $20 \mathrm{~dB}$ above the highest point of the noise floor. This can be a very serious problem when this tone is coupled down to the baseband by the clock noise, which will potentially destroy the performance of the modulator in audio applications, where the human ear is the end receiver.

The time domain estimation also confirms the advantages in tonal behavior of the AIB in consistency with the frequency domain. The short-term autocorrelation reveals that there is less periodicity in the AIB than that in the standard modulator. This is even more convincing a result because human ear is sensitive to short-term periodicity, which can not always be observed from the power spectrum.

\section{ACKNOWLEDGEMENT}

The authors wish to acknowledge the early support of this work from Texas Instruments Incorporated and Motorola Incorporated.

\section{REFERENCES}

[1] J.C. Candy, G.C. Temes, "Oversampling Methods for A/D and D/A Conversion", in Oversampling Delta-Sigma Data Converters, IEEE Press, 1992, pp. 1-29.

[2] S.R. Norsworthy, "Quantization Errors and Dithering in Delta-Sigma Modulators", in Delta-Sigma Data Converters, IEEE Press, 1997, pp. 75-140.

[3] R. M. Gray, "Quantization Noise in Delta-Sigma A/D Converters", in Delta-Sigma Data Converters, IEEE Press, 1997, pp. 44-74.

[4] S. R. Northworthy, "Effective Dithering of Sigma-Delta Modulators", IEEE ISCAS'92

[5] X. Sun and K.R. Laker, "Adaptive Integrator-Output Bounding (AIB) for Second Order Sigma-Delta ADC", IEEE ICSP' 00 .

[6] X. Sun and K.R. Laker, "A New Design for Cascaded SigmaDelta Modulatores", IEEE ISCAS'OI

[7] P. J. A. Naus and E. C. Dijkmans, "Low Signal-Level Distortion in Sigma-Delta Modulators," $84^{\text {th }}$ Convention of the Audio Engineering Society, preprint 2584(D-4) 